\title{
Time to update radiological criteria for non-invasive diagnosis of hepatocellular carcinoma
}

\author{
Sanjay Saini · Daniel S. Pratt
}

Received: 8 September 2014/Accepted: 21 September 2014/Published online: 2 November 2014

(C) Asian Pacific Association for the Study of the Liver 2014

Over the last 50 years remarkable progress has been made in the management of patients with chronic liver disease [1, 2]. This includes those patients who develop hepatocellular carcinoma (HCC), in part because early detection of $\mathrm{HCC}$ has been aided by advances in diagnostic imaging [3]. Liver transplantation offers the best outcome for early stage HCC in patients with cirrhosis [4]. Therefore, bridging therapies can be considered to those awaiting a donor liver [5].

Today, computed tomography (CT) scanning and magnetic resonance (MR) imaging are commonly employed for detecting and characterizing nodules in cirrhotic livers [6]. Due to heterogeneity in tumor biology, for lesions $1 \mathrm{~cm}$ and larger, strict $\mathrm{CT}$ and MR criteria are used for noninvasive diagnosis of $\mathrm{HCC}$ allowing patient prioritization for transplantation [7, 8]. For lesions not meeting these criteria, nodule biopsy or nodule growth during serial imaging studies can be employed for diagnosing HCC [79]. Despite increasing availability of high-resolution CT scanning and MR imaging, lesions under $1 \mathrm{~cm}$ are generally ignored because pathology studies have shown that the majority of these nodules detected on imaging in a setting of cirrhosis are not malignant [10].

Recently, the use of dual function, namely, extracellular plus hepatobiliary action, MR contrast agents have

\section{S. Saini $(\bowtie)$}

Department of Radiology, Massachusetts General Hospital and Harvard Medical School, 32 Fruit Street, Boston, MA 02114, USA

e-mail: ssaini@partners.org

\section{S. Pratt}

Department of Medicine, Massachusetts General Hospital and Harvard Medical School, 32 Fruit Street, Boston, MA 02114, USA been advocated for improved detection and characterization of cirrhotic nodules over that of traditional, extracellular-only MR contrast agents [11]. These dual-action contrast agents permit evaluation of tumor vascularity in the early dynamic imaging-phase and tumor uptake of the contrast agent via sodium-independent organic anion transporters in the delayed hepatocyte-phase [12]. In nodules not demonstrating the characteristic arterial enhancement followed by washout, the absence of tumor enhancement in the hepatocyte phase is also suggestive of HCC [13]. However, these criteria are only applied to lesions $1 \mathrm{~cm}$ and larger. Therefore an important challenge for imaging of the cirrhotic liver has been to determine management of sub-centimeter, hypointense nodules seen on hepatocyte-phase high resolution MR imaging. This is the problem Toyoda and colleagues investigated in their study published in this issue of Hepatology International [14].

Radiologists traditionally try to characterize each liver lesion individually, thus treating each nodule as an independent entity. However, in this study, the investigators took a novel approach. They studied patients who underwent HCC resection or HCC ablation with or without chemo-embolization to determine if patient clinical outcome was affected by the presence or absence of these hypointense sub-centimeter nodules in the surrounding liver. Their study showed that disease recurrence was in fact higher when these hypointense nodules were present.

There are three implications of this work.

First, these findings need to be confirmed in Asian and non-Asian subjects. This is important not only due to genetic differences in various populations, but also the well-established differences in the underlying causes of chronic liver disease in Asian, North American and European populations [15]. 
Second, if the observations are replicated, they portend important implications for HCC management options as these treatments are both expensive and have considerable associated morbidity. Specifically in patients with subcentimeter non-enhancing nodules on hepatocyte phase MR images, expedited transplantation might be a better option than resection, which in turns appears to be superior to ablative therapy [14].

Finally, and most importantly, the radiological criteria for non-invasive radiological diagnosis of HCC in patients with cirrhosis that permits the use of either CT scanning or MR imaging and relies on nodule vascularity should be updated to reflect these findings and those of numerous other publications that have investigated the efficacy of hepatobiliary MR contrast agents. There is an increasing body of evidence, although admittedly mostly from East Asia, that dual action MR contrast agents provide additional information that increases diagnostic sensitivity for detecting HCC [16]. Thus, not only would these findings, if replicated, suggest a preference for MR imaging over CT scanning, it would also suggest that there should be a preference for newer dual-action MR contrast agents over older extracellular-only contrast agents.

In conclusion, the investigators should be commended for their innovative study, which reinforces the view that current radiological criteria for non-invasive diagnosis of HCC should be updated to incorporate imaging with hepatocyte-specific contrast agents.

\section{References}

1. Chung RT, Baumert TF. Curing chronic hepatitis C: the arc of a medical triumph. N Engl J Med. 2014;370:1576-1578

2. Dienstag JL, Cosimi AB. Liver transplantation: a vision realized. N Engl J Med. 2012;367:1483-1485
3. Saini S. Imaging of the hepatobiliary tract. $\mathrm{N}$ Engl $\mathrm{J}$ Med. 1997;336:1889-1894

4. Bruix J, Sherman M. Management of hepatocellular carcinoma: an update. AASLD Practice Guideline 2010. http://www.aasld. org/practiceguidelines/Documents/Bookmarked\%20Practice $\% 20$ Guidelines/HCCUpdate2010.pdf. Accessed 13 October 2014

5. Belghiti J, Carr BI, Greig PD, Lencioni R, Poon RT. Treatment before liver transplantation for HCC. Ann Surg Oncol. 2008;15:993-1000

6. Kudo M. Multistep human hepatocarcinogenesis: correlation of imaging with pathology. Gasteroenterology. 2009;44:112-118

7. Bruix J, Sherman M. Management of hepatocellular carcinoma: an update. Hepatology. 2011;53:1020-1022

8. European Association For The Study Of The Liver. European organization for research and treatment of cancer. EASL-EORTC clinical practice guidelines: management of hepatocellular carcinoma. J Hepatol. 2012;56:908-943

9. Song P, Tobe RG, Inagaki Y, et al. The management of hepatocellular carcinoma around the world: a comparison of guidelines from 2001 to 2011. Liver Int. 2012;32:1053-1063

10. Roskams T. Anatomic pathology of hepatocellular carcinoma: impact on prognosis and response to therapy. Clin Liver Dis. 2011;15:245-259

11. Cruite I, Schroeder M, Merkle EM, Sirlin CB. Gadoxetate disodium-enhanced MRI of the liver: part 2, protocol optimization and lesion appearance in the cirrhotic liver. Am J Roentgenol. 2010;195:29-41

12. Narita M, Hatano E, Arizono S, et al. Expression of OATP1B3 determines uptake of Gd-EOB-DTPA in hepatocellular carcinoma. J Gasterenterol. 2009;44:793-798

13. Bartolozzi C, Battaglia V, Bargellini I, Bozzi E, Campani D, Pollina LE et al. Contrast-enhanced magnetic resonance imaging of 102 nodules in cirrhosis: correlation with histological findings on explanted livers. Abdom Imaging. 2013;38:290-296

14. Toyoda H, Kumada T, Tada T, Sone Y, Maeda A, Kaneoka Y. Non-hypervascular hypointense nodules on Gd-EOB-DTPA enhanced MRI as a predictor of outcomes for early-stage HCC. Hepatology International. 2015

15. Venooka AP, Papandreoub C, Furusec J, de Guevarad LL. The Incidence and epidemiology of hepatocellular carcinoma: a global and regional perspective. Oncologist. 2010;15:5-13

16. Van Beers BE, Pastor CM, Hussain HK. Primovist, Eovist: what to expect. J Hepatol. 2012;57:421-429 